\title{
Effets de deux types de déjections animales sur la croissance en longueur de deux variétés de concombre (Cucumis sativus)
}

\author{
KOUAKOU Kouassi Joseph ${ }^{1 *}$, YAO Koffi Bertin ${ }^{1}$, SIKA Ahoua Edmond², GOGBEU Seu \\ Jonathan ${ }^{3}$, KONÉ Loua Serge Patrick ${ }^{1}$, DOGBO Dénezon Odette ${ }^{1}$ \\ 1 Université Nangui Abrogoua, Unité de Formation et de Recherche des Sciences de la Nature, Laboratoire de \\ Biologie et Amélioration des Productions Végétales, 02 BP 801 Abidjan 02, Côte d'Ivoire \\ 2Université Nangui Abrogoua, Unité de Formation et de Recherche des Sciences et Gestion de l'Environnement, \\ 02 BP 801 Abidjan 02, Côte d'Ivoire \\ 3Université Jean Lorougnon Guédé, Laboratoire de Physiologie et Pathologie Végétales, BP 150 Daloa, Côte \\ d'Ivoire
}

*Auteur correspondant : ecologue12@yahoo.fr

Original submitted in on $18^{\text {th }}$ January 2019. Published online at www.m.elewa.org/journals/ on $30^{\text {th }}$ April 2019 https://dx.doi.org/10.4314/jab.v136i1.3

\section{RÉSUMÉ}

Objectif : L'objectif de l'étude est d'évaluer l'efficacité de la fiente de poulet et du lisier de porc sur la croissance en longueur de deux variétés de concombre, POINSETT et F1 TOKYO, les plus cultivées en Côte d'Ivoire.

Méthodologie et résultats : La couche superficielle du sol de la parcelle expérimentale de l'Université Nangui Abrogoua a été prélevée à $20 \mathrm{~cm}$ de profondeur, séchée et tamisée à $2 \mathrm{~mm}$. La partie fine recueillie a servi de substrat pour l'étude. Les différents fertilisants étudiés sont la fiente de poulet, le lisier de porc, le mélange fiente de poulet et lisier de porc et l'engrais minéral NPK. Des sachets en plastique ont servi de support pour les milieux de culture. Les doses croissantes de fiente de poulet ont retardé l'émergence des plantules des deux variétés. Les meilleurs résultats sur la croissance des plantes ont été obtenus avec le mélange de fertilisants.

Conclusion et application : Le mélange fiente de poulet et lisier de porc favorise plus la croissance en longueur des deux variétés de concombre. II peut être utilisé en amendement de sol pour la production des deux variétés de concombre.

Mots clés : concombre, variété POINSETT, variété F1 TOKYO, fiente de poulet, lisier de porc.

\begin{abstract}
Objective: The study objective is to evaluate the effectiveness of chicken manure and pig slurry on the growth in length of two varieties of cucumber, POINSETT and F1 TOKYO, most cultivated in Côte d'Ivoire.

Methodology and results: The soil surface layer of the experimental plot of Nangui Abrogoua University was taken at a depth of 20 centimeters, dried and sieved to two millimeters. The fine part collected served as a substrate for the study. The different fertilizers studied are poultry manure, pig slurry, poultry and slurry manure mix and NPK mineral fertilizer. Plastic bags were used as support for culture media. Increasing doses of chicken manure delayed the emergence of seedlings of both varieties. The best results on plant growth were obtained with the fertilizer mix.
\end{abstract}


Conclusion and application: The slurry mix of chicken and pig manure favors longer growth in both varieties of cucumber. It can be used as a soil amendment for the production of both varieties of cucumber.

Keywords: cucumber, POINSETT variety, TOKYO F1 variety, poultry manure, pig manure.

\section{INTRODUCTION}

En 2025, plus des deux-tiers de la population mondiale habitera en ville. Dans tous les pays du Sud, le processus d'urbanisation s'accélère, malgré les efforts de certains gouvernements de freiner l'exode rural. La concentration de la population dans les villes génère des besoins nouveaux en termes d'alimentation (Moustier, 2003) et l'agriculture urbaine est reconnue comme un enjeu majeur en termes d'approvisionnement des villes, d'emploi et de gestion de l'environnement urbain (Houot et al., 2003). Les besoins en denrées alimentaires sont corrélés avec une augmentation de la production de matières organiques telles que les déchets urbains (boues de station d'épuration, eaux domestiques usées), les effluents d'élevages et les sous-produits agro-industriels (De Bon et al., 2009). Ces déchets constituent une source d'éléments fertilisants. Dans le contexte d'insécurité alimentaire, de réduction de la

\section{MATÉRIEL ET MÉTHODES}

Site d'étude: L'étude a été menée à l'Université Nangui Abrogoua (UNA), située dans la ville d'Abidjan, au sud de la Côte d'lvoire. Les coordonnées géographiques du site d'étude sont de $5^{\circ} 17^{\prime}$ et $5^{\circ} 31^{\prime}$ de latitude nord et $3^{\circ} 45^{\prime}$ et $4^{\circ} 22^{\prime}$ de longitude ouest (Koffi et al,, 2009). La ville d'Abidjan est soumise à un climat de type subéquatorial comprenant quatre saisons, à savoir, une grande (mars à juillet) et une petite saison de pluie (octobre à novembre), une grande (décembre à février) et une fertilité des sols agricoles et de la hausse des prix des engrais sur les marchés, il apparaît nécessaire d'utiliser, pour l'agriculture, les nutriments disponibles et à faible coût (Useni et al., 2013). Selon les Nations Unies (2007), 40 à $60 \%$ de la population ivoirienne résident actuellement en ville et d'ici 2025 , ce nombre augmentera de $1 \%$ chaque année. La population urbaine a triplé en 23 ans (Kouakou et al., 2010). La Côte d'lvoire, comme beaucoup d'autres pays africains, a connu, ces dernières années, un développement rapide du maraîchage et de l'élevage urbains et périurbains (Kouakou et al., 2008; Kouakou et al., 2010). Le présent travail est une contribution à la valorisation agricole des déjections animales. II s'agira d'évaluer l'efficacité de la fiente de poulet et du lisier de porc sur la croissance en longueur de deux variétés de concombre.

petite sèche (août à septembre) (Kouakou et al., 2010) . Abidjan a un climat de type tropical et la température moyenne est de $26,6^{\circ} \mathrm{C}$. II tombe en moyenne $1784 \mathrm{~mm}$ de pluie par an et le sol est de type sablo-argileux (N'Dri, 2016).

Matériel végétal : Le matériel végétal est constitué des semences de deux variétés du cultivar Cucumis sativus, les plus cultivées en Côte d'Ivoire. Nous avons la variété pollen, nommée POINSETT, et la variété hybride, nommée F1 TOKYO (Figure 1). 


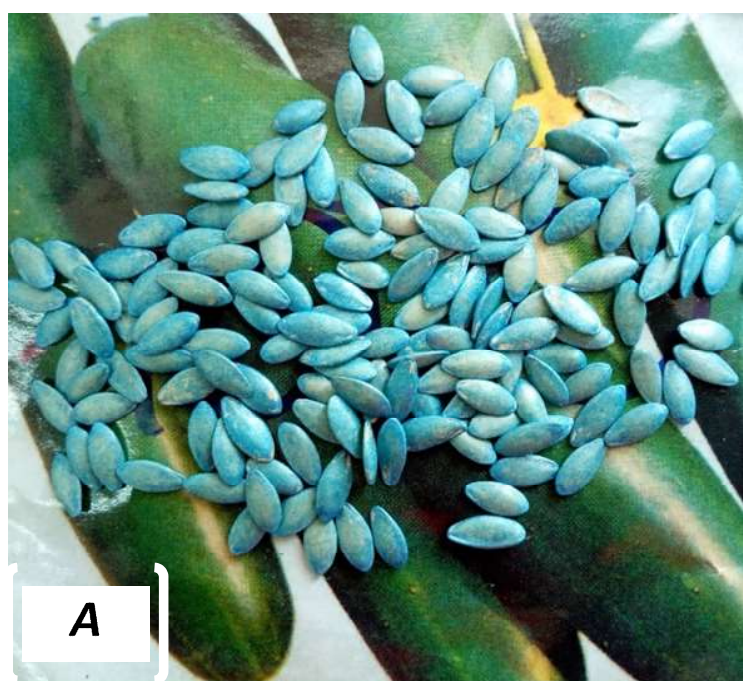

Figure 1 : Fruits et graines de F1 TOKYO $(\mathrm{A})$ et POINSETT $(\mathrm{B})$

Substrat des milieux de culture: Sur la parcelle expérimentale de l'UNA, la couche superficielle du sol a été prélevée à $20 \mathrm{~cm}$ de profondeur, séchée et tamisée à $2 \mathrm{~mm}$. La partie fine recueillie a servi de substrat pour l'étude.

Matériel fertilisant : Le matériel fertilisant comprend la fiente de poulet, le lisier de porc et l'engrais minéral NPK. La fiente de poulet et le lisier de porc proviennent, respectivement, d'une ferme avicole, dans la commune de Port-Bouët, et d'une ferme porcine, dans la commune de Bingerville.

Milieux de culture : Les milieux de culture pour notre travail sont représentés dans le tableau 1.La méthode de préparation des milieux a été inspirée de celles de

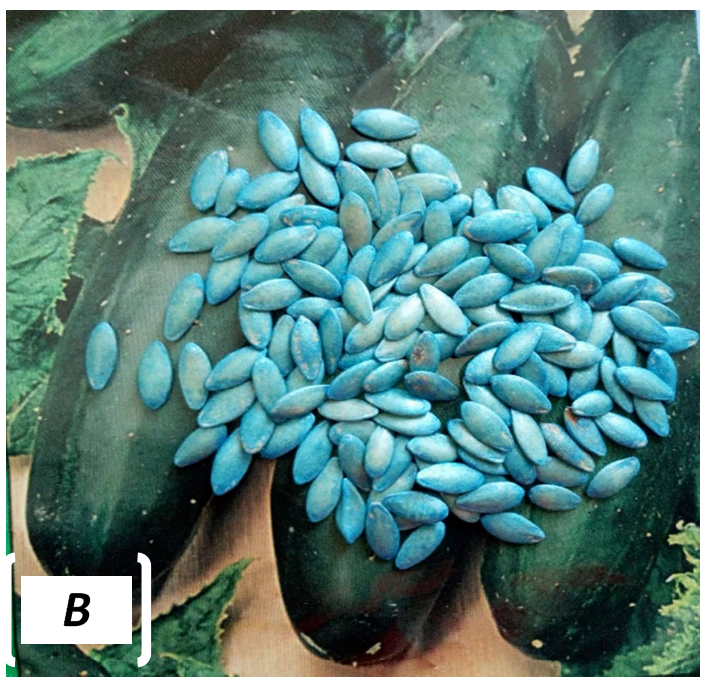

Coco sol (2017) et M'Sadak et al. (2013). Pour chaque dose et chaque fertilisant, dix répétitions ont été effectuées. Les milieux ont été arrosés pendant 14 jours afin de faciliter la décomposition des engrais organiques avant le semis.

Semis du concombre et entretien de la parcelle : Le semis des graines a été fait le 5 octobre 2017 dans des sachets en plastique (Figure 2) contenant le milieu de culture. Le tuteurage des plantes a été effectué dès l'apparition de la première vrille de la plante, en dressant une ficelle qui a servi de tuteur aux vrilles. Pendant la plantation, deux arrosages par jour (matin et soir) ont été effectués.

Tableau 1: Composition des différents milieux de culture

\begin{tabular}{l|l|l}
\hline Traitements & Composition des milieux de culture & $\begin{array}{l}\text { Dose ou rapport de la quantité } \\
\text { d'engrais organique sur la } \\
\text { quantité de sol }\end{array}$ \\
\hline Sol simple (Témoin) & $2 \mathrm{~kg}$ de sol & $0 \mathrm{~kg} / \mathrm{kg} \mathrm{de} \mathrm{sol}$ \\
\hline Sol + NPK & $2 \mathrm{~kg}$ de sol $+15 \mathrm{~g}$ de NPK $(12-22-22)$ & \\
\hline Substrat avec du fumier composé (fiente +lisier) & \\
\hline M1 & $2 \mathrm{~kg}$ de sol $+0,5 \mathrm{~kg}$ de mélange de fumiers & $0,25 \mathrm{~kg} / \mathrm{kg}$ de sol \\
\hline M2 & $2 \mathrm{~kg}$ de sol $+1 \mathrm{~kg}$ de mélange de fumiers & $0,5 \mathrm{~kg} / \mathrm{kg}$ de sol \\
\hline M3 & $2 \mathrm{~kg}$ de sol $+1,5 \mathrm{~kg}$ de mélange de fumiers & $0,75 \mathrm{~kg} / \mathrm{kg}$ de sol \\
\hline Substrat avec du fumier simple & \\
\hline F1 & $2 \mathrm{~kg}$ de sol $+0,5 \mathrm{~kg}$ de fiente de poulet & $0,25 \mathrm{~kg} / \mathrm{kg}$ de sol \\
\hline F2 & $2 \mathrm{~kg}$ de sol $+1 \mathrm{~kg}$ de fiente de poulet & $0,5 \mathrm{~kg} / \mathrm{kg}$ de sol \\
\hline F3 & $2 \mathrm{~kg}$ de sol $+1,5 \mathrm{~kg}$ de fiente de poulet & $0,75 \mathrm{~kg} / \mathrm{kg}$ de sol \\
\hline L1 & $2 \mathrm{~kg}$ de sol $+0,5 \mathrm{~kg}$ de lisier de porc & $0,25 \mathrm{~kg} / \mathrm{kg}$ de sol \\
\hline L2 & $2 \mathrm{~kg}$ de sol $+1 \mathrm{~kg}$ de lisier de porc & $0,5 \mathrm{~kg} / \mathrm{kg}$ de sol \\
\hline L3 & $2 \mathrm{~kg}$ de sol $+1,5 \mathrm{~kg}$ de lisier de porc & $0,75 \mathrm{~kg} / \mathrm{kg}$ de sol \\
\hline
\end{tabular}






Figure 2 : Jeune plantule de la variété F1 TOKYO

Des produits phytosanitaires tels que Cypercal 50 EC (insecticide) et Ivory 80wp (fongicide) ont été utilisés pour la protection des plantes. L'expérimentation a duré six (6) semaines après le semis.
Paramètres mesurés: Les paramètres végétatifs mesurés sont représentés dans le tableau 2.

Tableau 2 : Paramètres végétatifs mesurés

\begin{tabular}{l|l|l}
\hline Caractères mesurés & \multicolumn{1}{|c|}{ Caractéristiques } & Méthodes de mesure \\
\hline Taux d'émergence & $\begin{array}{l}\text { Apparition des feuilles cotylédonaires au- } \\
\text { dessus du sol }\end{array}$ & $\begin{array}{l}\text { Pourcentage du nombre de graines } \\
\text { présentant des feuilles } \\
\text { cotylédonaires sur le nombre total } \\
\text { de graines semées }\end{array}$ \\
\hline Longueur de la plante & Longueur de la plante durant sa croissance & Mesure de la tige principale \\
\hline
\end{tabular}

Analyse de données: Le logiciel STATISTICA version 7.1 a été utilisé pour l'analyse des données. Une comparaison des moyennes entre les différents traitements a été faite grâce à l'analyse de la variance (ANOVA). Pour un caractère donné, lorsqu'on

\section{RÉSULTATS}

Taux d'émergence des plantes: Les résultats relatifs au taux d'émergence des plantes, après semis, sont présentés dans le tableau 3. Trois jours après le semis, les résultats ont montré que pour la plupart les traitements, les graines ont donné un bon taux d'émergence, à l'exception des graines ayant pour milieux de culture la fiente à la dose $2(0,5$ observe une différence significative entre les traitements, on complète l'ANOVA par des comparaisons multiples en effectuant le test Tukey au seuil de $5 \%$.

$\mathrm{kg} / \mathrm{kg}$ de sol) et $3(0,75 \mathrm{~kg} / \mathrm{kg}$ de sol), chez les deux variétés. Pour ces graines, les premières émergences ont été observées 14 jours après le semis. Le résultat le plus intéressant est que le taux d'émergence diminue pour des doses croissantes de fiente de poulet.

Tableau 3 : Evaluation du taux d'émergence après le semis

\begin{tabular}{|c|c|c|c|c|}
\hline Variétés & Fertilisants & Doses & $\begin{array}{l}\text { Nombre moyen de } \\
\text { plantes émergées }\end{array}$ & $\begin{array}{l}\text { Pourcentage } \\
\text { d'émergence }\end{array}$ \\
\hline & \multirow{3}{*}{ Fiente } & $\overline{F 1}$ & $8 / 10$ & $80 \%$ \\
\hline & & F2 & $1 / 10$ & $10 \%$ \\
\hline & & F3 & $0 / 10$ & $0 \%$ \\
\hline & \multirow[b]{3}{*}{ Lisier } & $\overline{\mathrm{L} 1}$ & $10 / 10$ & $100 \%$ \\
\hline & & $\bar{L} 2$ & $10 / 10$ & $100 \%$ \\
\hline & & L3 & $10 / 10$ & $100 \%$ \\
\hline
\end{tabular}




\begin{tabular}{|c|c|c|c|c|}
\hline \multirow{5}{*}{ POINSETT } & \multirow{3}{*}{ Mélange } & $\mathrm{M} 1$ & $9 / 10$ & $90 \%$ \\
\hline & & M2 & $10 / 10$ & $100 \%$ \\
\hline & & M3 & $9 / 10$ & $90 \%$ \\
\hline & $\overline{N P K}$ & NPK & $10 / 10$ & $100 \%$ \\
\hline & Témoin & Témoin & $10 / 10$ & $100 \%$ \\
\hline \multirow{11}{*}{ F1 TOKYO } & \multirow{3}{*}{ Fiente } & $\mathrm{F} 1$ & $8 / 10$ & $80 \%$ \\
\hline & & F2 & $2 / 10$ & $20 \%$ \\
\hline & & F3 & $0 / 10$ & $0 \%$ \\
\hline & \multirow{3}{*}{ Lisier } & $\mathrm{L} 1$ & $8 / 10$ & $80 \%$ \\
\hline & & L2 & $9 / 10$ & $90 \%$ \\
\hline & & L3 & $10 / 10$ & $100 \%$ \\
\hline & \multirow{3}{*}{ Mélange } & M1 & $10 / 10$ & $100 \%$ \\
\hline & & $\mathrm{M} 2$ & $10 / 10$ & $100 \%$ \\
\hline & & M3 & $9 / 10$ & $100 \%$ \\
\hline & NPK & NPK & $10 / 10$ & $100 \%$ \\
\hline & Témoin & Témoin & $10 / 10$ & $100 \%$ \\
\hline
\end{tabular}

$\mathrm{F} 1$ : fiente de poulet à la dose $1 ; \mathrm{F} 2$ : fiente de poulet à la dose $2 ; \mathrm{F} 3:$ fiente de poulet à la dose $3 ; \mathrm{L} 1$ : lisier de porc à la dose $1 ; \mathrm{L} 2$ : lisier de porc à la dose $2 ; \mathrm{L} 3$ : lisier de porc à la dose $3 ; \mathrm{M} 1$ : mélange de fumiers à la dose $1 ; \mathrm{M} 2$ : mélange de fumiers à la dose $2 ; \mathrm{M} 3$ : mélange de fumiers à la dose 3

Effet de la variété de concombre sur la longueurs atteintes par les variétés de concombre croissance en longueur des plantes: Les sont de même ordre de grandeur (Tableau 4).

Tableau 4 : Longueur moyenne de la plante en fonction de la variété

\begin{tabular}{l|l}
\hline Variétés & Longueur moyenne de la plante $(\mathbf{c m})$ \\
\hline POINSETT & $118,1 \pm 46,5 \mathrm{a}$ \\
\hline F1 TOKYO & $119,8 \pm 43,1 \mathrm{a}$ \\
\hline $\mathrm{P}$ & 0,204 \\
\hline
\end{tabular}

Les chiffres suivis de la même lettre sont statistiquement identiques au seuil de $5 \%$.

Effet de l'interaction entre la variété et le fertilisant sur la croissance en longueur du concombre : Les résultats consignés dans le tableau
5 montrent que l'interaction entre la variété et le fertilisant influence significativement la croissance en longueur des plantes.

Tableau 5 : Longueur moyenne de la plante en fonction de l'interaction entre la variété et le fertilisant

\begin{tabular}{c|c|c}
\hline Variétés & Fertilisants & Longueur moyenne de la plante $\mathbf{( c m})$ \\
\hline \multirow{4}{*}{ POINSETT } & Fiente & $92,3 \pm 44,7 \mathbf{g}$ \\
\cline { 2 - 3 } & Lisier & $126,8 \pm 32,4 \mathbf{c}$ \\
\cline { 2 - 3 } & Mélange & $154,6 \pm 41 \mathbf{a}$ \\
\cline { 2 - 3 } & NPK & $97 \pm 9,9 \mathbf{f}$ \\
\cline { 2 - 3 } & Témoin & $58,6 \pm 6 \mathbf{i}$ \\
\cline { 2 - 3 } & Fiente & $127,7 \pm 56,6 \mathbf{c}$ \\
\cline { 2 - 3 } & Lisier & $103,3 \pm 25,5 \mathbf{e}$ \\
\cline { 2 - 3 } & Mélange & $143,9 \pm 30,3 \mathbf{b}$ \\
\cline { 2 - 3 } & NPK & $111,5 \pm 54,8 \mathbf{d}$ \\
\cline { 2 - 3 } & Témoin & $63,4 \pm 27,4 \mathbf{h}$ \\
\hline \multirow{4}{*}{ F1 TOKYO } & & 0,010048 \\
\hline
\end{tabular}

Les chiffres suivis de la même lettre sont statistiquement identiques au seuil de $5 \%$.

Chez les deux variétés de concombre, le mélange de fertilisants favorise plus la croissance en longueur des plantes. Cette croissance est plus importante chez la variété POINSETT. 
Effet de l'interaction entre la variété, le fertilisant

végétaux : Le tableau 6 renseigne sur la longueur et la dose sur la croissance en longueur des atteinte par les plantes.

Tableau 6 : Longueur moyenne de la plante en fonction de l'interaction entre la variété, le fertilisant et la dose

\begin{tabular}{|c|c|c|c|}
\hline Variétés & Fertilisants & Doses & $\begin{array}{l}\text { Longueur moyenne de la plante } \\
\text { (cm) }\end{array}$ \\
\hline \multirow{11}{*}{ POINSETT } & \multirow{3}{*}{ Fiente } & $\mathrm{D} 1$ & $117,7 \pm 32,4 \mathrm{e}$ \\
\hline & & D2 & $74 \pm 56,5 j$ \\
\hline & & D3 & $53,7 \pm 35,6 \mathbf{k l}$ \\
\hline & \multirow{3}{*}{ Lisier } & $\mathrm{D} 1$ & $89,2 \pm 23,8 \mathbf{i}$ \\
\hline & & D2 & $146,7 \pm 21,1 \mathrm{c}$ \\
\hline & & D3 & $139,5 \pm 15,4 d$ \\
\hline & \multirow{3}{*}{ Mélange } & $\mathrm{D} 1$ & $119,8 \pm 51,9 \mathbf{e}$ \\
\hline & & D2 & $177,4 \pm 32,3 a$ \\
\hline & & D3 & $157,1 \pm 33,9 b$ \\
\hline & NPK & NPK & $97 \pm 9,8 \mathbf{g}$ \\
\hline & Témoin & Témoin & $58,6 \pm 13,4 \mathrm{I}$ \\
\hline \multirow{12}{*}{ F1 TOKYO } & \multirow{3}{*}{ Fiente } & $\mathrm{D} 1$ & $157,1 \pm 22,7 \mathbf{b}$ \\
\hline & & $\mathrm{D} 2$ & $140 \pm 30,4 d$ \\
\hline & & D3 & $84 \mathrm{~h}$ \\
\hline & \multirow{3}{*}{ Lisier } & $\mathrm{D} 1$ & $85,3 \pm 21 \mathrm{hi}$ \\
\hline & & D2 & $100,2 \pm 20,8 \mathbf{g}$ \\
\hline & & D3 & $120,7 \pm 22,1 \mathrm{e}$ \\
\hline & \multirow{3}{*}{ Mélange } & $\mathrm{D} 1$ & $119,6 \pm 20,6 \mathbf{e}$ \\
\hline & & D2 & $148,4 \pm 25,6 \mathbf{c}$ \\
\hline & & D3 & $159,3 \pm 21,9 \mathbf{b}$ \\
\hline & NPK & NPK & $111,5 \pm 46,2 f$ \\
\hline & Témoin & Témoin & $63,4 \pm 10 k$ \\
\hline & $P$ & & 0,000012 \\
\hline
\end{tabular}

Les chiffres suivis de la même lettre sont statistiquement identiques au seuil de $5 \%$.

Les plantes les plus longues ont été observées avec le mélange de fertilisants à la dose 2, chez POINSETT $(177 \mathrm{~cm})$, et à la dose 3 , chez F1 TOKYO $(159,3 \mathrm{~cm})$.

Effet de l'interaction entre la variété et le fertilisant sur la croissance en longueur des plantes: Les résultats consignés dans le tableau 7 montrent que chez les deux variétés de concombre, les meilleures croissances ont été obtenues avec le mélange de fertilisants.

Tableau 7: Longueur moyenne atteinte par la plante en fonction de l'interaction entre la variété et le fertilisant

\begin{tabular}{c|c|c}
\hline Variétés & Fertilisants & Longueur moyenne de la plante $(\mathbf{c m})$ \\
\hline \multirow{4}{*}{ POINSETT } & Fiente & $92,3 \pm 44,7 \mathbf{f}$ \\
\cline { 2 - 3 } & Lisier & $126,8 \pm 32,4 \mathbf{c}$ \\
\cline { 2 - 3 } & Mélange & $154,6 \pm 40,9 \mathbf{a}$ \\
\cline { 2 - 3 } & NPK & $97 \pm 9,9 \mathbf{e}$ \\
\cline { 2 - 3 } & Témoin & $58,6 \pm 5,9 \mathbf{g}$ \\
\hline \multirow{4}{*}{ F1 TOKYO } & Fiente & $127,7 \pm 56,6 \mathbf{c}$ \\
& Lisier & $103,3 \pm 25,5 \mathbf{e}$ \\
\cline { 2 - 3 } & Mélange & $143,9 \pm 30,3 \mathbf{b}$ \\
\cline { 2 - 3 } & NPK & $11,5 \pm 54,8 \mathbf{d}$ \\
\cline { 2 - 3 } & Témoin & $63,4 \pm 27,4 \mathbf{g}$ \\
\hline
\end{tabular}

Les chiffres suivis de la même lettre sont statistiquement identiques au seuil de $5 \%$. 
Effet de l'interaction entre la variété, le fertilisant et la dose sur la croissance en longueur des végétaux: Le tableau 8 nous renseigne sur la longueur atteinte par les plantes en fonction des facteurs.

Tableau 8 : Longueur moyenne de la plante en fonction de l'interaction entre la variété, le fertilisant et la dose

\begin{tabular}{|c|c|c|c|}
\hline Variétés & Fertilisants & Doses & $\begin{array}{l}\text { Longueur moyenne de la plante } \\
\text { (cm) }\end{array}$ \\
\hline \multirow{11}{*}{ POINSETT } & \multirow{3}{*}{ Fiente } & $\overline{D 1}$ & $117,66 \pm 32,4 \mathrm{e}$ \\
\hline & & D2 & $74,00 \pm 56,5 j$ \\
\hline & & D3 & $53,66 \pm 35,61$ \\
\hline & \multirow{3}{*}{ Lisier } & $\overline{D 1}$ & $89,16 \pm 23,8 \mathrm{~h}$ \\
\hline & & D2 & $146,70 \pm 21,1 \mathrm{c}$ \\
\hline & & D3 & $139,50 \pm 15,4 d$ \\
\hline & \multirow{3}{*}{ Mélange } & $\mathrm{D} 1$ & $119,75 \pm 51,9 \mathrm{e}$ \\
\hline & & $\mathrm{D} 2$ & $177,40 \pm 32,3 a$ \\
\hline & & D3 & $157,10 \pm 33,9 b$ \\
\hline & NPK & NPK & $97 \pm 9,8 \mathrm{~g}$ \\
\hline & Témoin & Témoin & $58,62 \pm 13,4 \mathrm{k}$ \\
\hline \multirow{12}{*}{ F1 TOKYO } & \multirow{3}{*}{ Fiente } & $\mathrm{D} 1$ & $157,10 \pm 22,7 b$ \\
\hline & & $\mathrm{D} 2$ & $140 \pm 30,4 d$ \\
\hline & & D3 & $84 i$ \\
\hline & \multirow{3}{*}{ Lisier } & D1 & $85,33 \pm 21 \mathrm{i}$ \\
\hline & & $\mathrm{D} 2$ & $100,20 \pm 20,8 \mathrm{~g}$ \\
\hline & & D3 & $120,75 \pm 22,1 \mathrm{e}$ \\
\hline & \multirow{3}{*}{ Mélange } & D1 & $119,62 \pm 20,6 \mathrm{e}$ \\
\hline & & $\mathrm{D} 2$ & $148,40 \pm 25,6 \mathrm{c}$ \\
\hline & & D3 & $159,30 \pm 21,9 b$ \\
\hline & NPK & NPK & $111,5 \pm 46,2 f$ \\
\hline & Témoin & Témoin & $63,37 \pm 10 \mathrm{k}$ \\
\hline & $P$ & & 0,000012 \\
\hline
\end{tabular}

Les chiffres suivis de la même lettre sont statistiquement identiques au seuil de $5 \%$.

\section{DISCUSSION}

La diminution du taux d'émergence pour des doses croissantes de fiente de poulet pourrait être due à la libération excessive de nitrate phytotoxique lors de sa décomposition. Selon Gazeau (2012), il faut considérer la fiente de poulet comme un engrais organique azoté bien que la teneur en azote total soit de l'ordre de 3\%. Les travaux de Boughaba (2012) ont montré qu'un taux de nitrate élevé se révèle comme un facteur limitant à la croissance et au développement des plantes avec l'obtention d'un taux d'émergence très faible sur un milieu de culture composé de $70 \%$ de fiente de poulet. La meilleure croissance des plantes de concombre obtenue avec le mélange de fumiers s'expliquerait par l'association de deux engrais complémentaires. En effet, Dauda et al. (2009) et Kra (2002) ont rapporté que l'incorporation de la fiente de poulet, reconnue pour sa richesse en azote $(\mathrm{N})$, dans les différentes structures cellulaires (bases azotées, protéines de structures, protéines enzymatiques), est nécessaire à la mise en place des membranes. Selon Villio et al. (2001), la fiente de poulet libère progressivement les minéraux; ce qui assurerait leur disponibilité au moment du besoin effectif par la plante. Le lisier de porc est riche en phosphore, avec un pourcentage de l'ordre de 4,5 (IF2O, 2008) et environ $80 \%$ du phosphore total se trouve sous forme minérale; ce qui en fait un élément rapidement utilisable par la plante (Levasseur, 1998; Villio et al., 2001). On constate que les doses croissantes de fumier favorisent la croissance en longueur du concombre. Ce résultat est conforme à celui rapporté par Tchabi et al. (2012) qui ont démontré, dans une étude sur la laitue, que des doses croissantes de bouse de vache favorisent sa croissance. Mais, les doses supérieures 
à la dose optimale sont préjudiciables à la plante à

\section{CONCLUSION}

II ressort de notre étude que des doses croissantes de fiente de poulet impactent négativement sur l'émergence des plantules de concombre. Le mélange de la fiente de poulet et du lisier de porc permet d'obtenir une meilleure croissance des

\section{RÉFÉRENCES}

Boughaba R., 2012. Étude de la gestion et valorisation des fientes par le lombricompostage dans la willaya de Constantine. Mémoire de Master en Ecologie et Environnement. Faculté des Sciences de la Nature et de la Vie (Département de Biologie et Ecologie). Université de Mentouri Constantine d'Algerie.98 pages.

Coco sol, 2017. Fiche technique : Culture hydroponique du concombre sous abri.(http://cocosol-ci.com/CSFT_CONCO MBRE.pdf). Consulté le 5 août 2017.

Dauda S., Ajayi F. et Ndor E., 2009. Growth and yield of watermelon (Citrullus lanatus) as affected by poultry manure application. Electronic Journal of Environmental, Agricultural and food Chemistry 8(4): 305-311.

De Bon H., Parrot L., Moustier P., 2008. Sustainable urban agriculture in developing countries. $A$ review. Agronomy for Sustainable Development 30 : 21-32.

Gazeau G., 2012. Les fientes de volailles. Matières organiques, fiche $\mathrm{N}$ 19. Chambres d'Agriculture Provence-Alpes-Côte d'Azur. 2 pages.

Houot S., Francou C., Vergé-Leviel C., Michelin J., Bourgeois $S$. ., Linères $M$. ., Morel $P$. , Parnaudeau V., Le Bissonnais Y., Dignac M. F., Dumat C., Cheiab A., Poitrenaud M., 2003. Valeur agronomique et impacts environnements de composts d'origine urbaine : variation avec la nature $\mathrm{du}$ compost. Dossier de l'environnement de I'INRA 25 :107-124.

IF2O (Interprofession des fertilisants organiques de l'Ouest), 2006. Caractérisation des fertilisants organiques. 104 pages.

Koffi K.K., Anzara G., Malice M., Dje Y., Bertin P., Baudoin J. and Zoro Bi I., 2009. Morphological and allozyme variation in a collection of Lagenaria siceraria (Molina) Standl. From Côte d'Ivoire. Biotechnology, Agronomy, Society and Environment, 13 (2): $257-270$ cause de l'excès d'azote (Sou et al., 2007).

plantes de concombre. Ce fait a été attribué à la complémentarité des deux types de déjections animales, riches, respectivement, en azote et en phosphore.

Kouakou K.J., Bekro Y.A., Sika A.E., Baize D., Dogbo D.O., Bounakhla M., Zahry F., Macaigne P., 2008. Diagnostic d'une contamination par les éléments traces métalliques de l'épinard (Spinacia oleracea) cultivé sur des sols maraîchers de la ville d'Abidjan (Côte d'Ivoire) amendés avec de la fiente de volaille. European Journal of Sciences Research, 21 (3) : 471-487.

Kouakou Y.E., Koné B., Bonfoh B., Kientga S. M., N'Go Y.A., Savane I. et Cissé G., 2010. L'étalement urbain au péril des activités agro-pastorales à Abidjan. Vertigo 10, numéro 2. Consulté le 4 décembre 2018. URL: http://journals.openedition.org/vertigo /10066 ; DOI : 10.4000/vertigo.10066.

Kra K., 2002. Effet de différentes doses d'azote sur quelques paramètres de croissance et de production de deux morphotypes de pois de terre [Vigna subterranea (L.) Verdc. (Fabaceae)]. Mémoire de Maîtrise. Unité de Formation et de Recherche des Sciences de la Nature, Université d'Abobo Adjamé, Abidjan (Côte d'Ivoire). 28 pages.

Levasseur P., 1998. Composition des lisiers de porc, facteurs de variation et méthodes d'évaluation. Techni Porc, 21 (3) :19-25.

Moustier P., 2003. Comptes Rendus des Séances de I'Académie d'Agriculture de France, Approvisionnement vivrier des villes du Sud : les enjeux et questions d'une agriculture de proximité, Paris (France), 89 (4) : 58-59.

M'sadak Y., Hamdi W., et Zaalani C., 2013. Production et croissance des plants d'Acacia sur des substrats à base de tamisat de compost dans une pépinière hors sol. Revue Agriculture, 06 : 29-34.

Nations Unies, 2007. Une croissance urbaine forte qui doit davantage à la croissance démographique qu'à l'urbanisation.[En ligne] URL:http://www.isted.com/poleville/dynamique_urbaine/urba2.pdf, Consulté le 04 décembre 2018.

N'Dri A., 2016. Effet de quelques fertilisants sur le rendement en tubercules de Manioc var. 
Yacé [Manihot esculenta Crantz (Euphorbiaceae)] cultivé en Côte d'Ivoire. Mémoire de Master 1, Unité de Formation et de Recherche des Sciences de la Nature, Université Nangui Abrogoua, Abidjan (Côte d'lvoire). 31 pages.

Sou M., Yacouba H. et Mermoud A., 2007. Valorisation du pouvoir fertilisant des eaux usées en agriculture maraîchère. EIER, Ouagadougou, Burkina Fasso. 13 pages.

Tchabi V. I., Azocli D. et Biaou D. G., 2012. Effet de différentes doses de bouse de vache sur le rendement de la laitue (Lactuca sativa L.) à Tchatchou au Bénin. Int. J. Biol. Chem. Sci. 6 (6): 5078-5084.

Useni S.Y., Chukiyabo K.M., Tshomba K.J., Muyambo M.E., Kapalanga K.P., Ntumba N.F., Kasangij K.P., Kyungu K.A., Baboy L.L., Nyembo K.L., Mpundu M.M., 2013. Utilisation des déchets humains recyclés pour l'augmentation de la production du maïs (Zea mays L.) sur un ferralsol du sud-est de la RD Congo. Journal of Applied Biosciences 66: 5070-50811.

Villio M., Arrouays D., Deslais W., Daroussin J., Bissonais Y. et Clergeot D., 2001. Estimation des quantités de matière organique exogène nécessaires pour restaurer et entretenir les sols limoneux français à un niveau organique donné. Etude et Gestion des Sols, 8 (1): 47-63. 